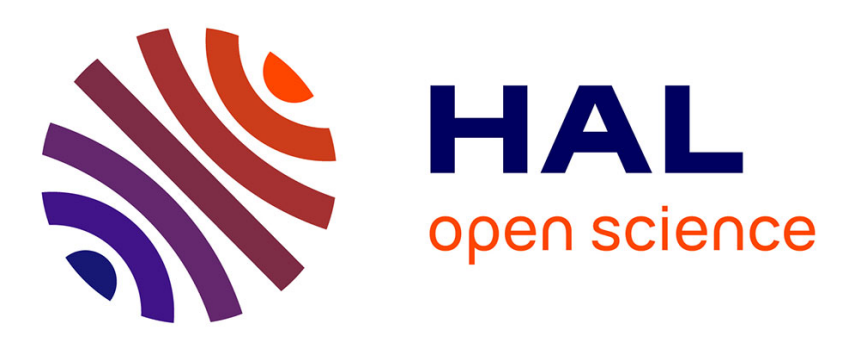

\title{
Les titanates de baryum dopés ont-ils des propriétés semiconductrices?
}

G. Godefroy, P. Jullien, L. Cai

\section{To cite this version:}

G. Godefroy, P. Jullien, L. Cai. Les titanates de baryum dopés ont-ils des propriétés semiconductrices?. Revue de Physique Appliquée, 1975, 10 (6), pp.399-402. 10.1051/rphysap:01975001006039900 . jpa-00243935

\section{HAL Id: jpa-00243935 https://hal.science/jpa-00243935}

Submitted on 1 Jan 1975

HAL is a multi-disciplinary open access archive for the deposit and dissemination of scientific research documents, whether they are published or not. The documents may come from teaching and research institutions in France or abroad, or from public or private research centers.
L'archive ouverte pluridisciplinaire HAL, est destinée au dépôt et à la diffusion de documents scientifiques de niveau recherche, publiés ou non, émanant des établissements d'enseignement et de recherche français ou étrangers, des laboratoires publics ou privés. 


\title{
LES TITANATES DE BARYUM DOPÉS ONT-ILS DES PROPRIÉTÉS SEMICONDUCTRICES ?
}

\author{
G. GODEFROY, P. JULLIEN et L. CAI
}

Laboratoire de Diélectriques et Ferroélectriques, Dijon, France

\begin{abstract}
Résumé. - On montre qu'un modèle à 2 niveaux d'impuretés, dans le titanate de baryum dopé au fer, au cobalt ou au cuivre, considéré comme un semi-conducteur à large bande interdite, permet d'expliquer qualitativement les courants et les photocourants observés. Les valeurs numériques des paramètres ne semblent pas être satisfaisantes.
\end{abstract}

Abstract. - A model with two impurity levels explains currents and photocurrents in $\mathrm{Fe}, \mathrm{Co}$, or $\mathrm{Cu}$ doped barium titanate, a large gap semiconductor.

1. Introduction. - Les titanates de baryum dopés au fer, au cobalt ou au cuivre peuvent être considérés comme des semiconducteurs à large bande interdite lorsqu'on se propose d'expliquer leur conductivité et leur photoconductivité. Il n'est cependant pas possible de linéariser les équations de bilan détaillé comme le font Bube [1], Rose [2] ou Ryvkin [3] car les photocourants ne sont jamais négligeables par rapport aux courants dans le noir, ils peuvent être jusqu'à 100 fois plus grands que les courants d'obscurité. Un modèle à deux niveaux d'impuretés peut être résolu en régime statique et traité numériquement en régime dynamique : on a donné au niveau profond le rôle de piège et au niveau proche de la bande de conduction, le rôle de donneur. La comparaison avec les résultats expérimentaux montre que le modèle proposé, s'il explique bien qualitativement la plupart des résultats, ne donne pas toujours des ordres de grandeurs corrects pour les paramètres introduits.

2. Le modèle à $\mathbf{2}$ niveaux. - Ce modèle a déjà été décrit dans [4] ; on rappelle les notations.

$E_{\mathrm{C}}$ énergie du bas de la bande de conduction de densité équivalente $N_{\mathrm{C}}$.

$E_{\mathrm{V}}$ énergie du haut de la bande de valence de densité équivalente $N_{\mathrm{V}}$.

$E_{M_{1}}$ énergie d'un niveau donneur photoexcitable de concentration $M_{1}$.

$E_{M_{2}}$ énergie d'un niveau de piège photoexcitable de concentration $M_{2}$.

2.1 DANS L'OBSCURITÉ, on trouve alors la densité de porteurs, en régime statique :

$$
n_{0}=\sqrt{M_{1} N_{\mathrm{C}}} \exp -\frac{E_{\mathrm{C}}-E_{M_{1}}}{2 k T} .
$$

2.2 SOUS ÉCLAIREMENT, AVEC DES PHOTONS D'ÉNERGIE $h v<h v_{\mathrm{D}}$ tels que

$$
h v_{\mathrm{D}}=E_{\mathrm{C}}-E_{M_{2}},
$$

seul le premier niveau est photoexcité ; on caractérise ce niveau par les paramètres $F_{1}$ et $B_{1}: F_{1}$ est la densité d'électrons excités par seconde optiquement à partir du niveau $E_{M_{1}}, B_{1}$ est la probabilité de recombinaison des électrons excités avec les impuretés de type $E_{M_{1}}$, la densité de porteurs $n_{i}$ en régime statique est :

$$
n_{i}=\sqrt{n_{0}^{2}+\frac{F_{1}}{B_{1}}} .
$$

Or, la vitesse de génération des porteurs est proportionnelle au coefficient d'absorption $\alpha$ et au nombre de photons arrivant sur l'unité de surface d'échantillon dans l'unité de temps :

$$
F_{1}=\eta_{1} \frac{\alpha \varphi}{h v}
$$

$\varphi$ : puissance lumineuse par unité de surface.

$\eta_{1}:$ rendement quantique ou rapport du nombre d'électrons créés au nombre de photons absorbés par le niveau 1.

Pour de faibles puissances, on peut écrire approximativement :

$$
n_{i} \sim n_{0}\left(1+\frac{\eta_{1} \alpha \varphi}{2 n_{0}^{2} h v B_{1}}\right) .
$$

Les régimes transitoires s'explicitent; la densité de porteurs $n$ s'écrit en fonction du temps : en début d'éclairement :

$$
n=n_{i} \operatorname{th} \frac{1}{2}\left(\frac{t}{\tau}+\gamma\right)
$$


où

$$
\tau=\frac{1}{2 B n_{i}}
$$

et

$$
\gamma=\log \frac{n_{i}+n_{0}}{n_{i}-n_{0}}
$$

en début d'obscurité :

$$
n=\frac{n_{0}}{\operatorname{th} \frac{1}{2}\left(\frac{t}{\tau^{\prime}}+\gamma\right)}
$$

où

$$
\tau^{\prime}=\frac{1}{2 B n_{0}} .
$$

2.3 SOUS ÉCLAIREMENT AVEC DES PHOTONS D'ÉNERGIE $h v>h v_{\mathrm{D}}$, le deuxième niveau est photoexcité ; il est caractérisé par les paramètres $F_{2}$ et $B_{2}$.

Le régime permanent donne une densité de porteurs $n_{i}^{\prime}$ sous éclairement :

$$
n_{i}^{\prime}=\sqrt{n_{0}^{2}+\frac{F_{1}}{B_{1}}+\frac{F_{2}}{B_{2}}} .
$$

Le flux lumineux $\varphi$ est alors lié aux paramètres $F_{1}$ et $F_{2}$ par la relation :

$$
F_{1}+F_{2}=\frac{\alpha \varphi}{h v} \frac{\eta_{1} M_{1}+\eta_{2} M_{2}}{M_{1}+M_{2}}
$$

si on fait l'hypothèse d'une probabilité d'absorption des photons par les impuretés 1 et 2 proportionnelles aux concentrations $M_{1}$ et $M_{2}$ de ces impuretés.

Sous faible éclairement, on a approximativement :

$n_{i}^{\prime} \sim n_{0}\left[1+\frac{\alpha \varphi}{2 n_{0}^{2} h v\left(M_{1}+M_{2}\right)}\left(\frac{\eta_{1}}{B_{1}} \frac{M_{1}}{B_{2}}+\frac{\eta_{2} M_{2}}{B_{2}}\right)\right]$.

L'étude du régime transitoire ne peut se faire que numériquement; néanmoins on peut donner les lois approchées suivantes :

- en début de régime transitoire,

$$
\left|\frac{\mathrm{d} n}{\mathrm{~d} t}\right|_{t=0} \simeq F_{1}+F_{2}
$$

- en fin de régime transitoire, les courbes calculées numériquement deviennent asymptotes aux courbes représentant les lois

- sous illumination :

$$
\log \frac{n_{i}+n}{n_{i}-n}=2 B n_{i}^{\prime} t+\text { Cte }
$$

- après illumination :

$$
\log \frac{n_{0}+n}{n_{0}-n}=2 B n_{0} t+\mathrm{Cte}
$$

où $B$ est approximativement la plus petite des 2 valeurs $B_{1}$ et $B_{2}$.

3. Les résultats de conductivité et de photoconductivité pour les titanates dopés. - 3.1 RÉGIME STATIQUE. - Les résultats, en régime statique, pour les titanates dopés au fer et au cobalt ont été donnés dans [4].

Dans le cas des cristaux dopés au cuivre, les variations de la conductivité et de la photoconductivité avec la température suivent une loi exponentielle :

$$
\sigma=\sigma_{0} \exp -\frac{W}{k T}
$$

comme les cristaux dopés au fer ou au cobalt.

La figure 1 montre les différentes valeurs trouvées pour $W$ en phase ferroélectrique ou paraélectrique, dans le noir ou sous éclairement par une lampe à vapeur de mercure ; on constate que l'énergie d'activation sous éclairement est plus faible que celle dans le noir, comme on peut le prévoir à partir de la valeur de $n_{i}^{\prime}$ en remplaçant $n_{0}$ par sa valeur.

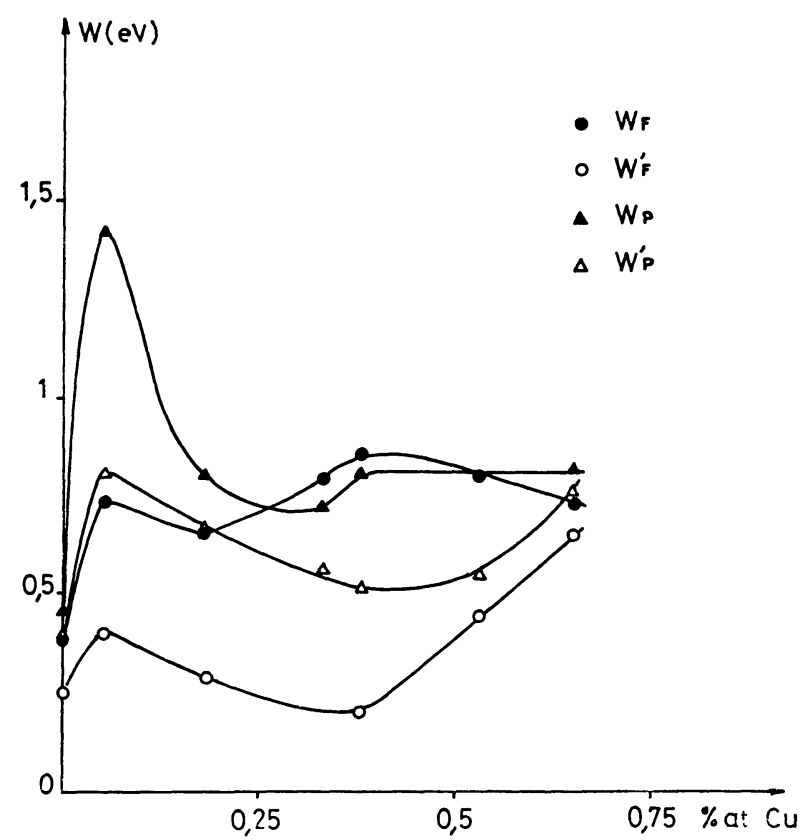

FIG. 1. - Variation de l'énergie d'activation avec le pourcentage en cuivre. $W_{F}=$ dans le noir, phase ferroélectrique. $W_{\mathrm{F}}^{\prime}=$ éclairement, phase ferroélectrique. $W_{\mathrm{P}}=$ dans le noir, phase paraélectrique. $W_{\mathbf{P}}^{\prime}=$ éclairement, phase paraélectrique.

La valeur de l'énergie d'activation est de l'ordre de $0,7 \mathrm{eV}$; ceci fixe la profondeur du niveau donneur à $1,4 \mathrm{eV}$. On remarque un maximum de $W$ pour une concentration de $0,05 \%$ at en $\mathrm{Cu}$ : ceci avait été observé pour le cobalt et relié au changement de mécanisme de compensation du dopant : les premiers 
résultats de dosage dans les cristaux dopés au cuivre semblent confirmer ce phénomène.

La figure 2 montre la variation de $\sigma_{0}$ avec la concentration; la figure 3 précise le rapport du courant sous éclairement au courant dans le noir pour différentes concentrations ; cette photosensitivité est plus grande pour les matériaux peu dopés et varie avec la température.
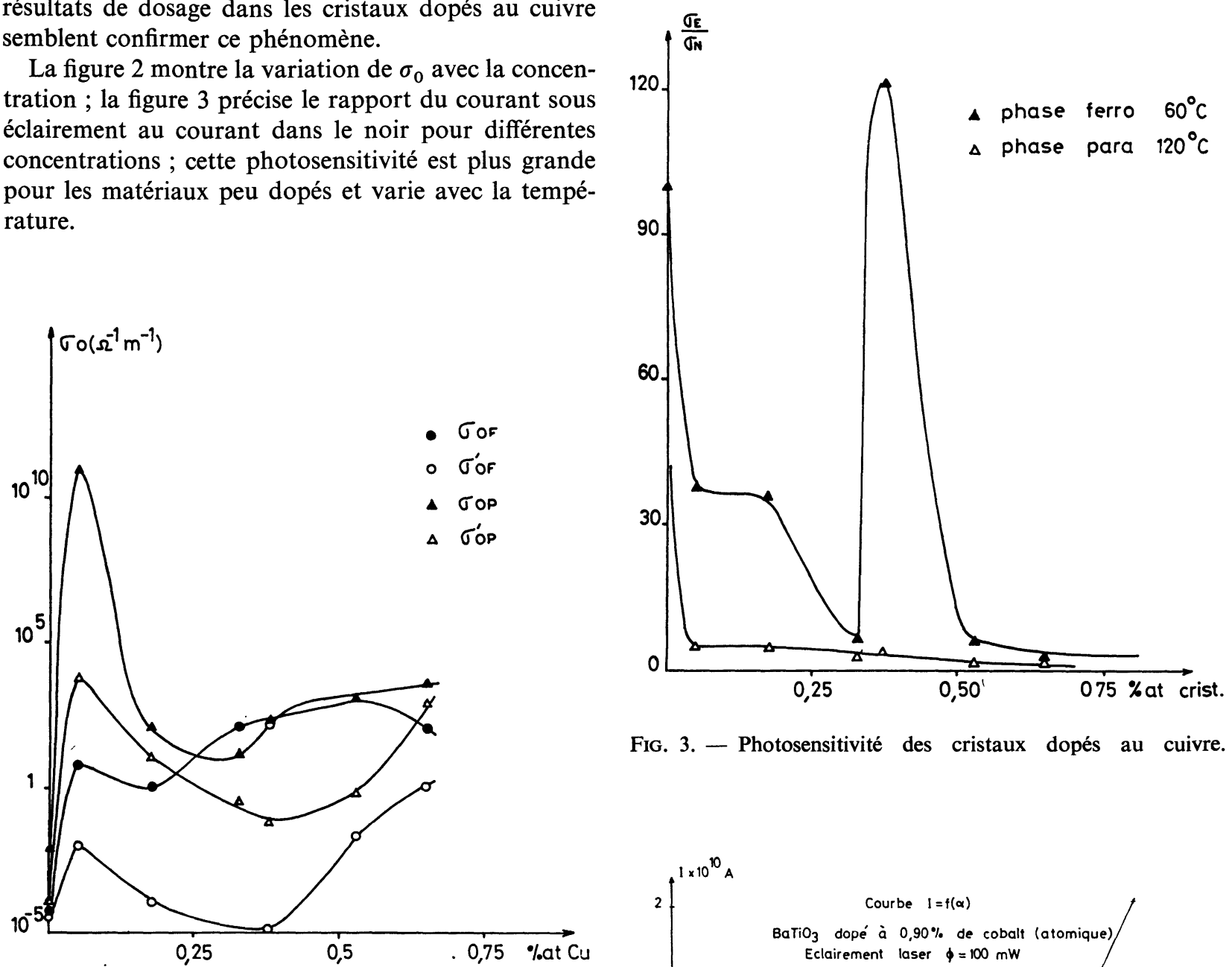

Fig. 3. - Photosensitivité des cristaux dopés au cuivre.

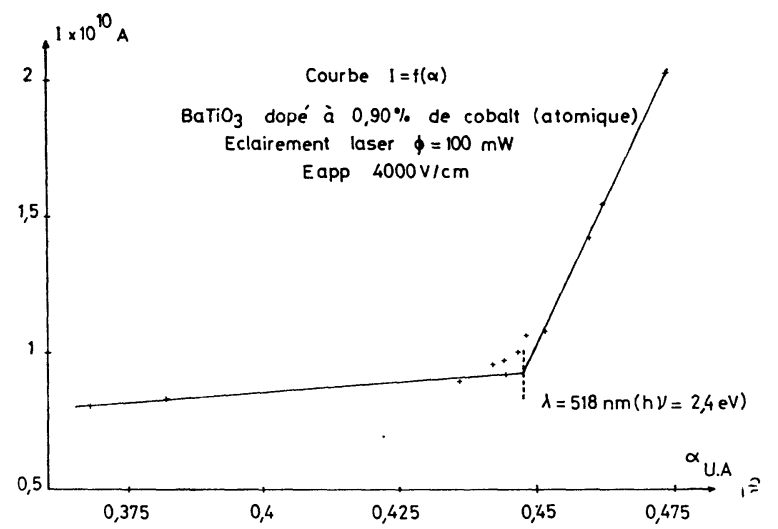

L'étude des photocourants sous éclairement laser d'intensité constante avec des longueurs d'onde variables montre sur la figure 4 que la variation de courant avec le coefficient d'absorption est formée de 2 portions de droite avec une discontinuité de pente pour $=518 \mathrm{~mm}$ soit une énergie $h v_{\mathrm{D}}=2,4 \mathrm{eV}$; on peut ainsi fixer la position du niveau de piège à 2,4 et au-dessous de la bande de conduction dans le cas des cristaux dopés au cobalt.

Par contre, les cristaux dopés au cuivre montrent 2 discontinuités $\lambda_{1}$ et $\lambda_{2}$, qui peuvent correspondre à deux niveaux de piège distincts.

3.2 RÉGIME TRANSITOIRE. - Les premières mesures en régime transitoire ont été faites pour des cristaux dopés au cobalt : la figure 5 montre la croissance des courants et la figure 6 leur décroissance.

Des courbes de décroissance, en fin de régime, l'étude théorique montre qu'on peut déduire la plus

FIG. 4. - Variation du photocourant avec absorption (cristaux dopés au cobalt).

petite valeur de $B$. Si on admet une mobilité de porteurs $\mu \sim 10^{-4} \mathrm{~m}^{2} \mathrm{~V}^{-1} \mathrm{~s}^{-1}$, comme dans les cristaux réduits [5], on trouve, pour toutes les longueurs d'onde :

$$
B \sim 1,5 \times 10^{-17} \mathrm{~m}^{3} \mathrm{~s}^{-1}
$$

cette valeur semble vraiment faible, car elle donnerait une valeur de section de capture de l'ordre de $10^{-22} \mathrm{~m}^{2}$, beaucoup trop petite.

Les pentes à l'origine des 2 régimes transitoires, $\beta_{\mathrm{e}}$ en début d'éclairement, $\beta_{0}$ en début d'obscurité après éclairement sont : 
$\lambda$ en $\AA$

$\beta_{\mathrm{e}}\left(\overline{\mathrm{As}}^{-1}\right)$

$\beta_{0}\left(\mathrm{As}^{-1}\right)$

$1,0 \times 10^{-10}$
$1,4 \times 10^{-10}$

$5,3 \times 10^{-11}$
$5,0 \times 10^{-11}$

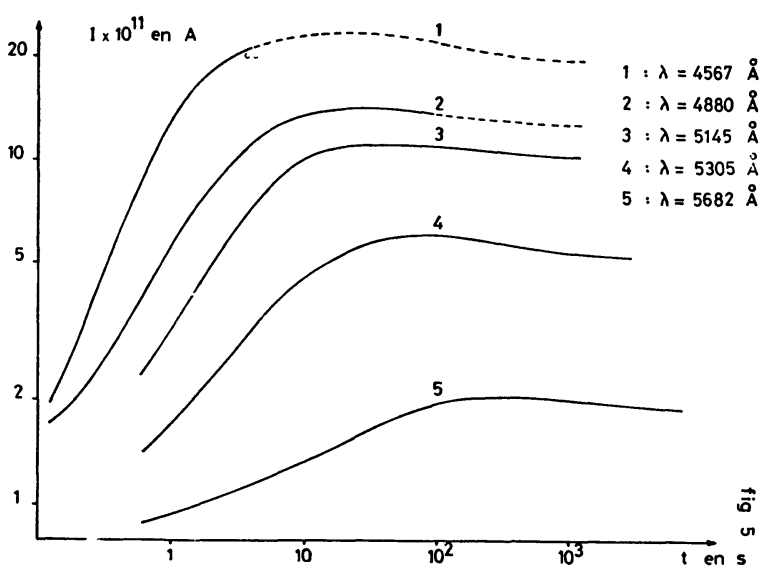

Fig. 5. - Régime transitoire sous éclairement (échelles logarithmiques) : cristaux au cobalt.

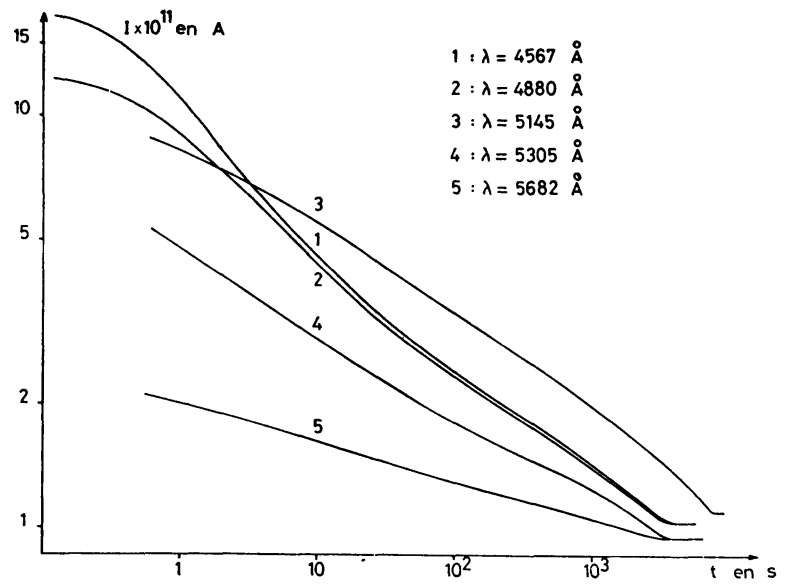

Fig. 6. - Régime transitoire après éclairement (échelles logarithmiques): cristaux au cobalt.

Compte tenu de la précision avec laquelle nous déterminons la valeur de ces pentes, nous pouvons admettre que pour chaque longueur d'onde du rayon-

\section{5}

$1,8 \times 10^{-11}$

$1,8 \times 10^{-11}$

$$
\begin{aligned}
& 8,0 \times 10^{-12} \\
& 9,1 \times 10^{-12}
\end{aligned}
$$$$
1,2 \times 10^{-12}
$$$$
1,3 \times 10^{-12}
$$

nement incident les pentes à l'origine des deux régimes transitoires sont les mêmes.

L'étude théorique montre que ces pentes représentent la valeur de la quantité $F_{1}+F_{2}$. Avec la valeur choisie pour la mobilité, on trouve :

$$
F_{1}+F_{2} \sim 10^{19} \mathrm{~m}^{-3} \mathrm{~s}^{-1} \text { pour } \lambda=5145 \AA .
$$

Cette valeur est supérieure à celle utilisée dans les résolutions numériques. De plus, on n'arrive pas à montrer que les variations de $F_{1}+F_{2}$ avec $\lambda$ sont liées à celles du coefficient d'absorption.

4. Discussion. - Le modèle proposé, dérivé de celui utilisé par les semiconducteurs, rend compte de l'existence de l'énergie d'activation $W$, telle que

$$
W=\frac{E_{\mathrm{C}}-E_{M}}{2},
$$

et de sa diminution sous éclairement, de la variation linéaire du photocourant avec le coefficient d'absorption avec une discontinuité de pente pour $\gamma_{D}$ telle que $h v_{\mathrm{D}}=E_{\mathrm{C}}-E_{M_{2}}$. Il permet d'expliquer l'allure des régimes transitoires.

Cependant certaines valeurs numériques sont surprenantes : celle du maximum de l'énergie d'activation, celles de $B_{1}$ et de $F_{1}+F_{2}$. De plus le comportement du régime transitoire sous éclairement est curieux : présence d'un faible maximum avant l'attente $\mathrm{du}$ régime stationnaire.

Il en résulte que l'on peut mettre en doute le comportement du titanate de baryum, comme un semiconducteur à large bande interdite. Des études complémentaires sont entreprises actuellement pour essayer de comprendre dans quelle mesure il serait souhaitable de tenir compte des phénomènes d'injection par les électrodes, des mouvements d'ions sous champ électrique, des effets photoferroélectriques, d'un mécanisme de conduction par polarons comme le suggèrent Reik et Heese [6] ou Berglund et Baer [7].

\section{Bibliographie}

[1] BuBE, R. H., Photoconductivity of solids (John Wiley and Sons, New York, London) 1966.

[2] Rose, A., traduit par SCHOTT : photoconduction, modeles et problemes annexes (Dunod) 1966.

[3] Ryvkin, S. M., Photoelectric effects in semi-conductors (Consultant Bureau, New York) 1964.
[4] Godefroy, G., Cochet, C., CaI, L., Jullien, P., J. Physique $36(1975) 137$.

[5] Kawabe, K. et Inuishi, Y., Japan J. Appl. Phys. 2 (1963) 590.

[6] Reik, H. et Heese, D., Phys. Stat. Sol. 24 (1967) 281.

[7] Berglund, C. and Baer, W., Phys. Rev. 1572 (1967) 358. 